\title{
Spike Timing Amplifies the Effect of Electric Fields on Neurons: Implications for Endogenous Field Effects
}

\author{
Thomas Radman, Yuzhuo Su, Je Hi An, Lucas C. Parra, ${ }^{\star}$ Marom Bikson ${ }^{\star}$ \\ Department of Biomedical Engineering, City College of the City University of New York, New York, New York 10031
}

\begin{abstract}
Despite compelling phenomenological evidence that small electric fields $(<5 \mathrm{mV} / \mathrm{mm})$ can affect brain function, a quantitative and experimentally verified theory is currently lacking. Here we demonstrate a novel mechanism by which the nonlinear properties of single neurons "amplify" the effect of small electric fields: when concurrent to suprathreshold synaptic input, small electric fields can have significant effects on spike timing. For low-frequency fields, our theory predicts a linear dependency of spike timing changes on field strength. For high-frequency fields (relative to the synaptic input), the theory predicts coherent firing, with mean firing phase and coherence each increasing monotonically with field strength. Importantly, in both cases, the effects of fields on spike timing are amplified with decreasing synaptic input slope and increased cell susceptibility (millivolt membrane polarization per field amplitude). We confirmed these predictions experimentally using CA1 hippocampal neurons in vitro exposed to static (direct current) and oscillating (alternating current) uniform electric fields. In addition, we develop a robust method to quantify cell susceptibility using spike timing. Our results provide a precise mechanism for a functional role of endogenous field oscillations (e.g., gamma) in brain function and introduce a framework for considering the effects of environmental fields and design of low-intensity therapeutic neurostimulation technologies.
\end{abstract}

Key words: electromagnetic field; hippocampus; electrical stimulation; frequency; gamma; coherence

\section{Introduction}

The study of how small electric fields affect brain function is important for several reasons. First, these studies provide insight into the mechanisms by which endogenous electric fields generated by the brain itself (e.g., delta, theta, gamma) could "feedback" unto the brain (Lutz et al., 2001, Parra and Bikson, 2004; Schaefer et al., 2006). Second, these studies address concerns about human exposure to environmental electromagnetic fields (Hamblin, 2002; Jefferys et al., 2003). Last, they have practical applications in the design of low-intensity brain stimulation treatments for neurological diseases (Ghai et al., 2000; Francis et al., 2003; Webster et al., 2006).

Small electric fields will polarize neurons by only a small amount; for this reason, small electric fields have been suggested previously to have no physiologically relevant effects. However, the hypothesis that small fields can affect brain function has garnered support from phenomenological studies applying lowintensity electrical stimulation to brain slices (Ghai et al., 2000;

\footnotetext{
Received Jan. 9, 2007; revised Feb. 9, 2007; accepted Feb. 12, 2007.

This work was supported in part by the Wallace H. Coulter Foundation and Professional Staff Congree-City University of New York. We thank Abhishek Datta of the City College of New York (New York, NY), Chris Leonard of New York Medical College (Valhalla, NY), Denis Paré of Rutgers University (Newark, NJ), Jack Belgum, Adair Osterle, and Rick Ayer (Sutter Instruments, Novato, CA), and Tim Bergel and Simon Gray (Cambridge Electronic Design, Cambridge, UK).

L.C.P. and M.B. contributed equally to this work.

Correspondence should be addressed to Marom Bikson, T-403B, Steinmann Hall, Department of Biomedical Engineering, City College of New York, 140th Street and Convent Avenue, New York, NY 10031. E-mail: bikson@ccny.cuny.edu.

DOI:10.1523/JNEUROSCI.0095-07.2007

Copyright $\odot 2007$ Society for Neuroscience $\quad$ 0270-6474/07/273030-07\$15.00/0
}

Francis et al., 2003; Bikson et al., 2004) and humans (Marshall et al., 2006; Webster et al., 2006), the latter indeed showing a causal effect on slow-wave oscillations and declarative memory. A quantitative and experimentally verified explanation for these findings has previously been lacking.

Although neurons often encode information in their firing rate, the timing of individual action potentials (APs) (temporal coding) has also been shown to carry significant information (de Ruyter et al., 1997). Cortical neurons have been identified that fire with an accuracy of a few milliseconds in response to sensory stimuli (Mainen and Sejnowski, 1996; Trussell, 1999; Kara et al., 2000; Reinagel and Reid, 2000; DeWeese et al., 2003), in synchrony with overt behavior (Riehle et al., 2000), and in-phase with ongoing extracellular potential oscillations (Kashiwadani et al., 1999; Mehta et al., 2002; Harris et al., 2003).

Here we consider how small electric fields, which are in themselves not sufficient to trigger or suppress action potential activation in response to synaptic input, may nonetheless have an effect on neuronal information processing through induced changes in spike timing. Specifically, given a steady firing threshold and knowing that a membrane polarizes linearly with field strength (in which the proportionality constant represents the susceptibility of the membrane potential to extracellular fields) (Bikson et al., 2004), we make a number of quantitative predictions on the effects of extracellular fields on spike timing of a neuron: (1) for relatively low-frequency or direct current (DC) fields, spike timing advances linearly with increasingly depolarizing field strength; (2) oscillating fields promote coherent firing with the mean phase of firing falling within less than one-quarter of the oscillatory cycle (on the positive rising edge); and (3) time ad- 
vancement, phase delay, and coherence strength all increase with field strength amplified by membrane susceptibility and the inverse synaptic ramp slope. These quantitative predictions are validated here for hippocampal CA1 neurons in vitro. In addition, we develop a method to determine membrane susceptibility based on spike timing measurements. Together, this work yields the first quantitative framework for evaluating the effect of subthreshold fields, with arbitrary (nonuniform) spatiotemporal waveform, on neuronal spike timing.

Parts of this work have been published previously in preliminary form (Radman et al., 2006).

\section{Materials and Methods}

Transverse hippocampal slices $(350 \mu \mathrm{m})$ were prepared from male Sprague Dawley rats (125-150 g), which were anesthetized with intraperitoneal ketamine $(7.4 \mathrm{mg} / \mathrm{kg})$ and xylazine $(0.7 \mathrm{mg} / \mathrm{kg})$ and killed by cervical dislocation. The slices were stored in a holding chamber submerged in artificial CSF (ACSF) consisting of the following (in mM): 125 $\mathrm{NaCl}, 26 \mathrm{NaHCO} 3,3 \mathrm{KCl}, 1.6 \mathrm{CaCl} 2,1.5 \mathrm{MgS0} 4,1.25 \mathrm{NaH}_{2} \mathrm{PO}_{4}$, and 10 glucose (bubbled with a mixture of $95 \% \mathrm{O}_{2}-5 \% \mathrm{CO}_{2}$ ). After $>60 \mathrm{~min}$, slices were transferred to an interface recording chamber at $33^{\circ} \mathrm{C}$.

Uniform electric fields were generated across individual slices by passing current between two parallel $\mathrm{Ag} / \mathrm{AgCl}$ electrodes (Ghai et al., 2000; Bikson et al., 2004) placed on the surface of the ACSF in the interface chamber; the wires were parallel to the direction of perfusate flow and were $12 \mathrm{~mm}$ long and $10 \mathrm{~mm}$ apart. Field intensities were generated by a Power 1401 analog-to-digital converter (Cambridge Electronic Design, Cambridge, UK) and converted to a constant current by a stimulus isolation unit (2200; A-M Systems, Carlsborg, WA). The electric field (in millivolts per millimeter) in the chamber was measured by two recording electrodes separated by $1 \mathrm{~mm}$ and calibrated to the current passed through the Ag- $\mathrm{AgCl}$ electrodes (Ghai et al., 2000; Durand and Bikson, 2001; Bikson et al., 2004); the polarity convention used refers to the anode on the alveus side of the hippocampus.

Conventional recording techniques were used to measure activity from the CA1 pyramidal cell region. Intracellular electrodes (40-120 $\mathrm{M} \Omega$, pulled on a P-97; Sutter Instruments, Novato, CA) were filled with $3 \mathrm{M}$ potassium chloride. Pyramidal neurons with an input resistance $>15.4 \mathrm{M} \Omega$, resting membrane potential (RMP) less than $-53.5 \mathrm{mV}$, overshooting action potentials, and no spontaneous action potentials were accepted. Cell input resistance was monitored with a $-0.3 \mathrm{nA}$ current step. The voltage recorded by a field electrode (placed within $50 \mu \mathrm{m}$ of the impaled neuron) was subtracted from the intracellular potential to obtain the transmembrane voltage and (partially) compensate for the exogenous potential artifact. For DC electric field experiments, no holding current was used. For alternating current (AC) electric field experiments, holding current (less than $-0.3 \mathrm{nA}$ ) was modulated to stabilize the RMP.

Depolarizing intracellular current ramps $(0.1-1.6 \mathrm{nA} / \mathrm{s})$ were generated and triggered to halt $<100 \mathrm{~ms}$ after action potential detection. The initiation of subsequent current ramps was intercalated by an $8 \mathrm{~s}$ delay. The experimental paradigm consisted of interlacing control (no field) ramp trials with ramps applied during field application. Extracellular field application was initiated $400 \mathrm{~ms}$ before activation of depolarizing intracellular current ramps. Alternate DC polarity fields or alternate 30 $\mathrm{Hz}$ AC fields shifted $1 / 60 \mathrm{~ms}\left(180^{\circ}\right)$ were tested, at varied peak field strengths (in millivolts per millimeter). AC stimulation also included interleaved DC field and control trials as required to determine membrane susceptibility (see Results). Signals were subtracted, amplified, and low-pass filtered $(1-10 \mathrm{kHz})$ with an Axoclamp-2B (Molecular Devices, Palo Alto, CA) and FLA-01 amplifiers (Cygnus Technology, Delaware Water Gap, PA) and then digitized and processed using a Power 1401 and Signal software (Cambridge Electronic Design).

Unless otherwise stated, all results are reported as means $\pm \mathrm{SD}$, and $n$ indicates number of cells. For resolution of lower-limit $(<5 \mathrm{mV} / \mathrm{mm})$ DC field effects, a paired $t$ test was performed for each cell at a single field magnitude by averaging time to first spike of neighboring control trials and pairing this with interlaced field trials (i.e., control/field/control), and positive and negative fields were grouped attributable to field-effect symmetry, resulting in 44-160 trials per neuron. For AC fields, phaseshifted trials of a single magnitude were combined (by subtracting $180^{\circ}$ from one), resulting in 11-164 trials per neuron and field condition. The phase of the first spike relative to the applied field was determined. Mean firing phase, $\Phi$, and vector strength, $r$, were computed from the population vector, which is defined as,

$$
\vec{r}=\int d \varphi p(\varphi) \vec{r}_{0}(\varphi)
$$

with $\vec{r}_{0}(\varphi)=[\cos \varphi, \sin \varphi]$, and $p(\varphi)$ is the distribution of phases. This population vector can be estimated from the observed phases $\varphi_{i}$ as the sample average,

$$
\vec{r}=\frac{1}{N} \sum_{i=1}^{N} \vec{r}_{0}\left(\varphi_{i}\right) .
$$

Linear regression was used to model the dependence of mean phase and coherence on field strength coefficient. Residuals from the regression line were used as criterion for outlier rejection based on interquartile range (Moore and McCabe, 1999).

\section{Results}

The single neuron amplification mechanism was validated using hippocampal CA1 pyramidal neurons in vitro. We first confirmed that firing time in response to injected depolarizing current ramps varies linearly with incremental polarization and in proportion to the inverse of ramp slope. We then demonstrated that timing changes in direct response to applied uniform DC fields follow the same linear properties. These timing changes were used to derive membrane polarization susceptibility. Finally, we confirmed the predicated effects of AC fields (in the gamma frequency range) on firing coherence and phase.

\section{Firing time changes linearly with increasing polarization}

Assuming a constant firing threshold, one would expect that an incremental polarization $\Delta V$, which is applied with a voltage ramp, $\dot{V}=d V / d T$, should lead to a time advancement of $\Delta t=$ $\Delta V / \dot{V}$, where $\Delta t$ is the firing time of control minus the field stimulus condition. Hyperpolarization of CA1 pyramidal neurons, with an increasingly negative DC holding current, $\Delta I$, incrementally delayed action potential firing time in response to an intracellular current ramp, $\dot{I}$. (Linear current ramps lead to linear voltage ramps if the change is sufficiently slow, here $0.1-1.6 \mathrm{nA} /$ s). Importantly, for the conditions tested here, action potential threshold did not vary with ramp slope or polarization, in agreement with previous findings (Fricker et al., 1999). The change in timing increased linearly with the holding current and was inversely proportional to ramp slope (Fig. 1$)\left(n=12\right.$ cells; $r^{2}>0.95$ for linear regressions). These results show that the membrane dynamics of real CA1 pyramidal neurons support the single neuron amplification mechanism hypothesized ( $R$ is membrane resistance)

$$
\Delta t=\frac{\Delta I}{\dot{I}}=\frac{R \Delta I}{R \dot{I}}=\frac{\Delta V}{\dot{V}} .
$$

Spike time advances with increasing applied DC electric fields Uniform DC electric fields were generated across hippocampal slices, whereas synaptic input was simulated by injecting a current ramp. Constant uniform fields induced a membrane polarization, consistent with previous reports (Chan and Nicholson, 


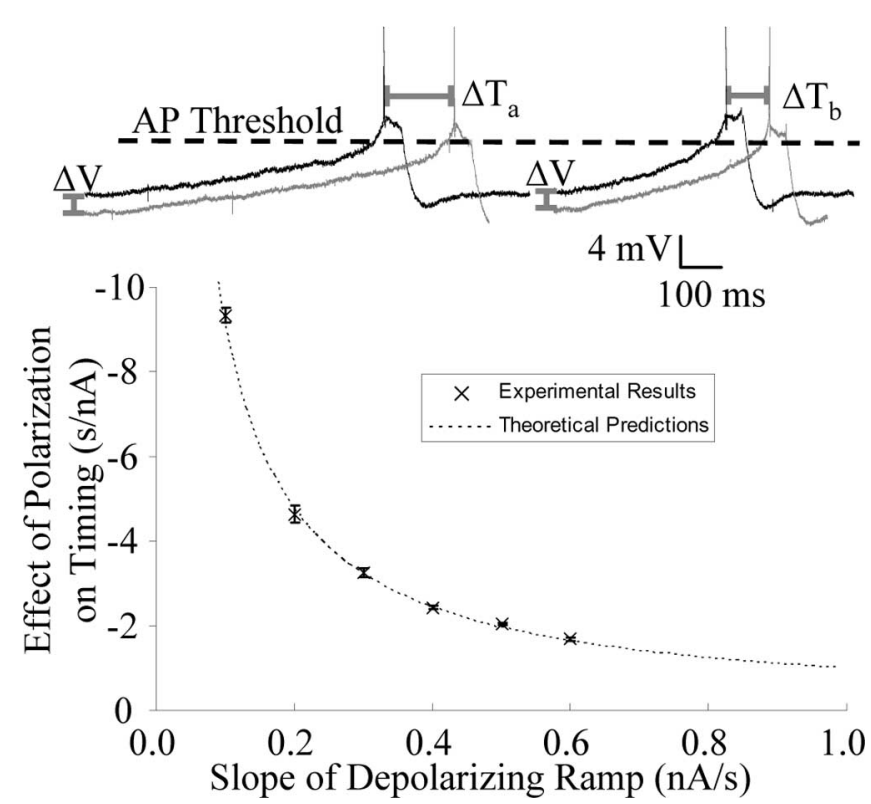

Figure 1. CA1 pyramidal cell response to depolarizing current ramps. Top, Intracellular recording of the response to a $0.4 \mathrm{nA} / \mathrm{s}$ (left) and $0.7 \mathrm{nA} / \mathrm{s}$ (right) intracellular current ramp (action potentials clipped). In both cases, hyperpolarization $(\Delta V)$ delayed the AP firing time. The change $(\Delta T)$ is inversely proportional to depolarizing ramp slope, $\Delta T_{\mathrm{a}}: \Delta T_{\mathrm{b}}$ as $1 / 0.4: 1 / 0.7$. Bottom, The inverse relationship between the ramp slope and the sensitivity to membrane polarization attributable to negative $D C$ holding current can be summarized in a single plot. Experimental results ( $\times$ symbols) fit directly to the theoretical prediction of a inverse slope. Error bars represent $\mathrm{SE}$ of the slope of a linear regression line fitting holding current (nanoamperes) versus AP timing (seconds) data ( $n \geq 7$ for linear regression data).

1986; Bikson et al., 2004), the magnitude of the somatic polarization was a linear function of field strength, and the polarity was dependent on the direction of the field (data not shown). Applied fields could significantly modulate the firing latency of single neurons resulting from intracellular depolarizing ramp current injection. DC fields inducing membrane hyperpolarization delayed action potential initiation, whereas fields inducing membrane depolarization had the opposite effect (Fig. 2A) $(n=8)$. For each injected ramp slope, the experimental data could be fit with a regression line $\left(r^{2}=0.90 \pm 0.1\right)$; the slope of this fit indicated the change in timing induced per millivolts per millimeter applied electric field (in $\mathrm{s} \cdot \mathrm{mV}^{-1} \cdot \mathrm{mm}^{-1}$ ). As intracellular ramp slope decreased, the fit timing sensitivity to applied fields $\left(\mathrm{s} \cdot \mathrm{mV}^{-1} \cdot \mathrm{mm}^{-1}\right)$ linearly increased, supporting our prediction of an inverse relationship. This inverse relationship is summarized for three cells in Figure $2 B$ in which, for each cell, the changes in time per electric field $(\Delta t / E)$ is plotted against the corresponding injected ramp slope $(\dot{V})$.

The somatic polarization induced by an electric field is set by the cell-specific membrane susceptibility constant $c, \Delta V=c E$. Membrane susceptibility can be determined using timing data by extension of Equation 1: $c=\dot{V} \Delta t / E$ (see Discussion, Eq. 2). Susceptibility may thus be calculated from a single timing change measurement at one field amplitude, using a single intracellular ramp slope. A more robust method involves using the inverse relationship in Figure $2 B$ to simultaneously fit all data from a single cell. Indeed, for each cell, these plots are well approximated by the curve $y=c x^{-1}\left(r^{2}>0.98 ; y=\Delta t / E ; x=\dot{V}\right)$, where $c$ indicates the estimated membrane susceptibility. Using this indicator, we determined polarization susceptibilities of $c=0.11 \pm$ $0.05 \mathrm{mV} / \mathrm{mV} \cdot \mathrm{mm}^{-1}(n=10)$. Using a conventional method (Bikson et al., 2004) consisting of recording the intracellular po-
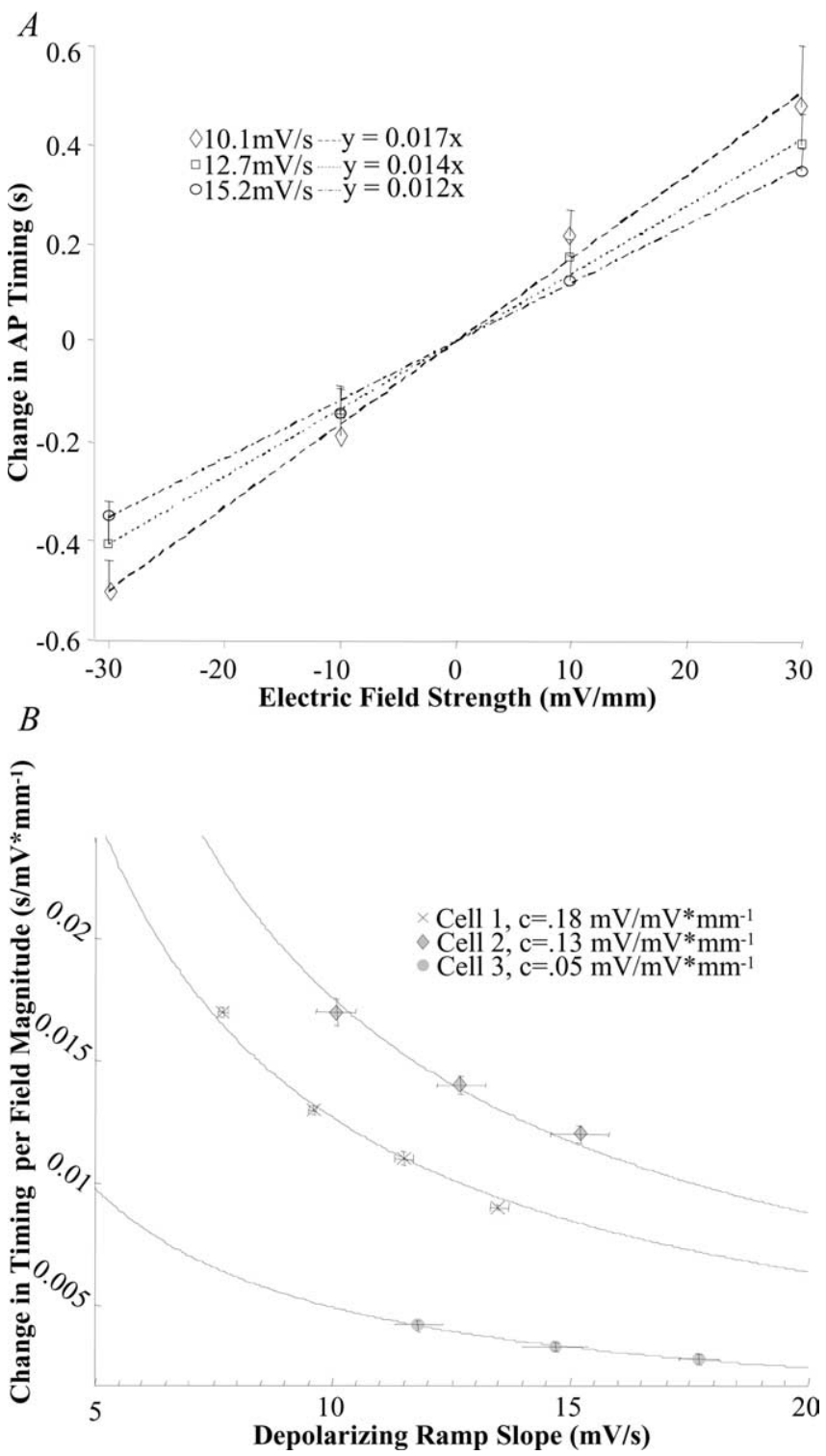

Figure 2. Effects of applied extracellular DC electric fields on CA1 pyramidal neuron firing time in response to intracellular depolarizing ramp slopes. $A$, Positive (hyperpolarizing) fields delayed action potential initiation, whereas negative (depolarizing) fields expedited AP onset. Note that, for each ramp slope series, the relationship between firing time $(\Delta t)$ and applied field amplitude is linear. Moreover, the slope of this relationship varies inversely with the slope of the injected intracellular ramp (i.e., 10.1:12.7:15.2 as 1/.017:1/.015:1/.012). The injected current ramp slope $(0.4,0.5,0.6 \mathrm{nA} / \mathrm{s})$ translated to voltage slope by cell resistance $(25.3 \mathrm{M} \Omega$ for this cell). The change in timing for any given field is "amplified" as the slope of the ramp is decreased. Reported are the mean \pm SD ( $r^{2}>0.94 ; p<0.01$ for the 3 regressions shown here). $\boldsymbol{B}$, The relationship between ramp slope and timing change per field magnitude is plotted for three cells. Each line in $\boldsymbol{A}$ corresponds to a point of the "Cell 3 " series shown (vertical error bars show SE of the slope, and the horizontal error bars show SEM). Data for each cell is fit with $y=$ $c / x\left(r^{2}>0.98 ; p<0.01\right.$ for all regressions shown). Legend shows membrane susceptibility, $c$, in units of $\mathrm{mV} / \mathrm{mV} \cdot \mathrm{mm}^{-1}\left(y \cdot x\right.$ or $\left.\mathrm{s} / \mathrm{mV} \cdot \mathrm{mm}^{-1} \cdot \mathrm{mV} / \mathrm{s}\right)$.

tential and subtracting the measured stimulation "artifact" at a second isopotential electrode, the polarization induced per electric field was $0.13 \pm 0.14 \mathrm{mV} / \mathrm{mV} \cdot \mathrm{mm}^{-1}(n=9)$.

\section{Resolution of lower-limit DC effects}

For a $0.4 \mathrm{nA} / \mathrm{s}$ injected current ramp, we observed a significant change in timing induced by $\pm 1 \mathrm{mV} / \mathrm{mm}$ DC field strengths $(n=$ 4). The average change in timing observed was $11 \pm 5 \mathrm{~ms}$ 
A

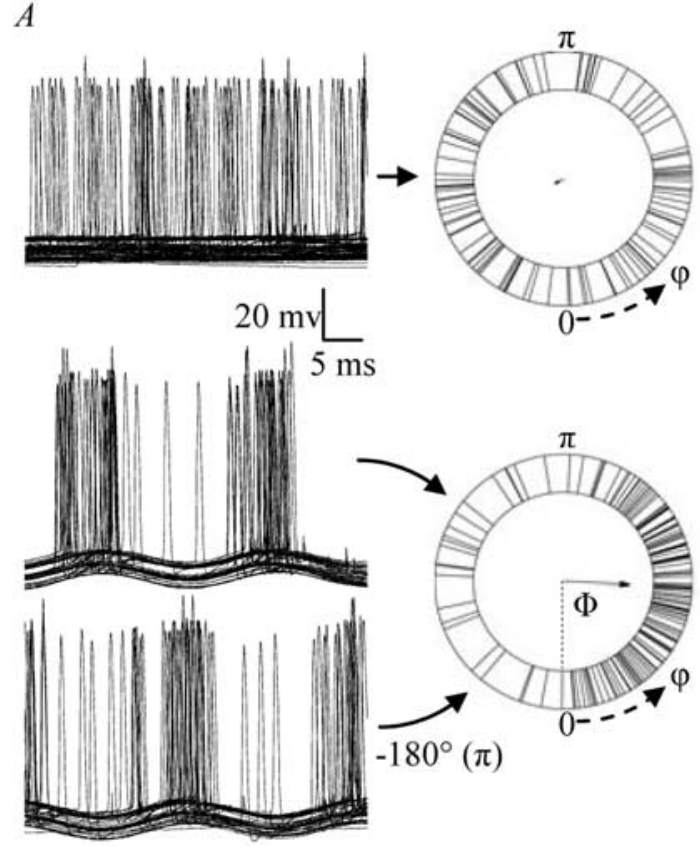

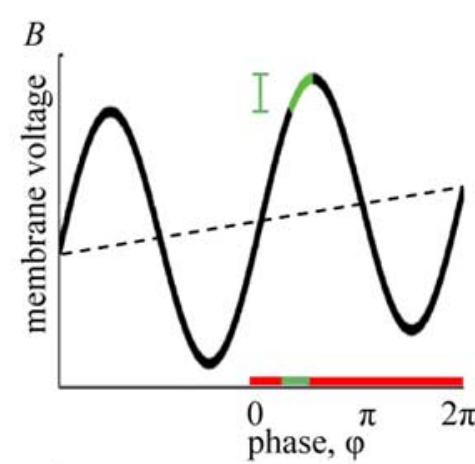

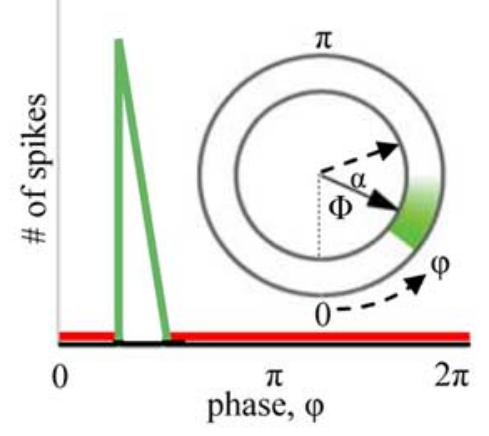

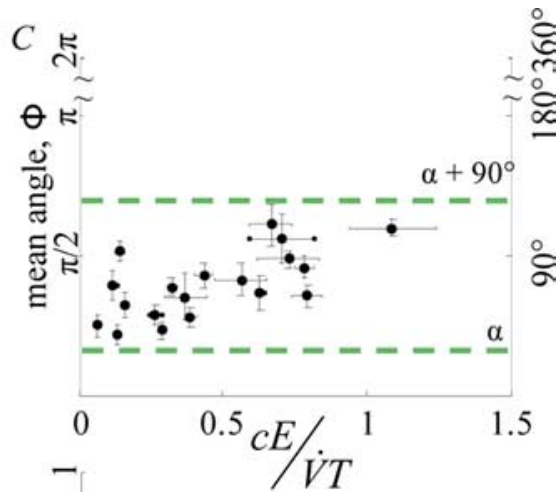

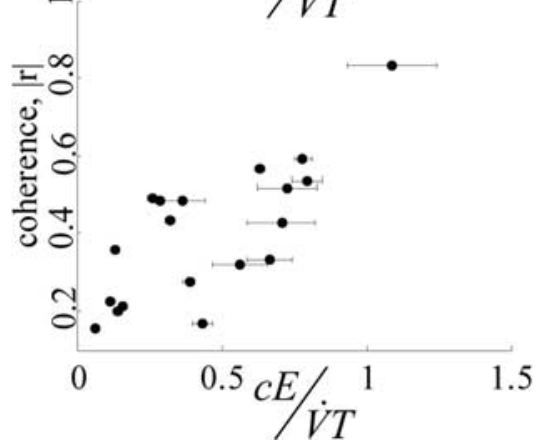

Figure 3. Effects of applied extracellular AC electric fields on CA1 pyramidal neuron firing time in response to intracellular depolarizing ramp slope. $A$, Overdrawn intracellular recordings in response to an injected current ramp with accompanying circular scatter plot. Top traces and circular plot, In the absence of uniform AC electric field (control), spike traces show low coherence and insignificant (random) mean angle. For circular scatter plots, outer edge shows individual spike phase $\varphi$, direction of arrow on inset shows mean angle $\Phi$, and length of arrow shows coherence as measured by vector strength $r$, with perfect coherence reaching edge of inset. Bottom traces and plot, Middle trace shows overdrawn samples in the presence of a $10 \mathrm{mV} / \mathrm{mm}$ uniform $30 \mathrm{~Hz} \mathrm{AC}$ electric field (stimulus), and bottom trace shows overdrawn samples in the presence of a $10 \mathrm{mV} / \mathrm{mm}$ uniform $30 \mathrm{~Hz} \mathrm{AC} \mathrm{electric} \mathrm{field} \mathrm{phase} \mathrm{shifted} 180^{\circ}$ from previous (stimulus). Bottom circular scatter plot combines field-present cases (by shifting data of bottom left case back $180^{\circ}$ ), and arrow shows increased coherence with a mean firing phase at $\sim \pi / 2$, on the rising edge of electric field oscillation. $\boldsymbol{B}$, Theoretical predictions of AP phase response to injected current ramp under AC electric field. Top, Dashed line shows simulated membrane voltage with same control condition as in $\boldsymbol{A}$. Black trace shows the simulated membrane response under field stimulus condition as in $\boldsymbol{A}$. If the previous cycle did not cross threshold, the membrane will not increase to a greater value during the red region on the phase axis. The cell is thus constrained to fire in the "unvisited" voltages (green), in this case on the positive rising field edge quadrant ( $<\pi / 2$ ). Bottom, Predicted distribution of spike timing phase; the inset shows the resulting circular plot of this distribution with mean angle and vector strength. Assuming a delay, $\alpha$, between the extracellular field and induced membrane polarization, the dashed arrow shows the mean firing phase relative to the extracellular field; note similarity to experimental results in $\boldsymbol{A}$. $\boldsymbol{C}$, Experimental results of mean phase and coherence vector strength. Horizontal axes is given by the experimentally observed value of $c \times E \times \dot{V}^{-1} \times T^{-1}$ (see Discussion). The dashed green lines indicate the field positive rising edge quadrant shifted by an estimated $\alpha=40^{\circ}$ membrane phase delay (see Discussion). Error bars show SEM.

(mean \pm SEM). Assuming a susceptibility of $0.11 \mathrm{mV}$ per 1 $\mathrm{mV} / \mathrm{mm}$ applied field and a $12 \mathrm{mV} / \mathrm{s}$ voltage ramp (induced by a $0.4 \mathrm{nA} / \mathrm{s}$ current injection into an $\sim 30 \mathrm{M} \Omega$ cell resistance), a change in timing of $\sim 9 \mathrm{~ms}$ would be expected.

For $0.5 \mathrm{mV} / \mathrm{mm}$ DC fields and a $0.4 \mathrm{nA} / \mathrm{s}$ current ramp, a statistically significant change in timing was observed in one of four neurons tested. For the cell showing a significant change, the average change in timing observed was $8 \pm 3 \mathrm{~ms}$ (mean $\pm \mathrm{SEM}$ ), whereas $4.5 \mathrm{~ms}$ was the predicted change (as above).

In attempts to resolve significant timing changes attributable to $0.5 \mathrm{mV} / \mathrm{mm}$ DC electric fields, we could reject the null hypothesis of a sample mean different than 0 in one of four attempts. For our experimental paradigm, the SD of the difference between time to action potential under a $0.5 \mathrm{mV} / \mathrm{mm}$ field, and a control trial was $54 \mathrm{~ms}$ for $0.4 \mathrm{nA} / \mathrm{s}$ current injections. If this $\mathrm{SD}$ remained stable over time, we would need $>500$ tests to resolve the predicted $4.5 \mathrm{~ms}$ difference induced by a $0.5 \mathrm{mV} / \mathrm{mm}$ field in $50 \%$ of neurons tested. Conversely, our attempts to resolve the effects of $0.5 \mathrm{mV} / \mathrm{mm}$ fields with $<160$ trials leads to a power of a $<80 \%$ chance to reject an effect when it is really there (type II, $\beta$ error); indeed, we rejected $75 \%$ of our attempts. For the predicted timing change in response to a $1 \mathrm{mV} / \mathrm{mm}$ field, we would expect to detect a significant change in $83 \%$ of our attempts $(<160$ trials); we resolved all four neurons. We note that the limits to resolve a field-induced change are determined by the noise (experimental and biological) in spike timing and subsequent effect on the repeats necessary to reach statistical significance; the latter is limited by duration of neuronal impalement integrity.

\section{Uniform AC electric fields induce spike coherence}

Uniform AC electric fields were generated across hippocampal slices, and the firing time in response to an intracellular depolarizing ramp was monitored. The frequency $(30 \mathrm{~Hz})$ and slope of the $\operatorname{ramp}(72 \pm 22 \mathrm{mV} / \mathrm{s})$ were selected to mimic extracellular gamma oscillations and synaptic theta depolarization (Harris et al., 2002) typical for theta-modulated gamma activity (Chrobak et al., 2000, Fischer et al., 2002). Significant coherence of the firing time with the applied field oscillation was observed in 21 of 29 field conditions (Rayleigh test, $p<0.02$ ), including fields as low as $1 \mathrm{mV} / \mathrm{mm}$. Mean firing phase was analyzed for 19 field conditions (excluding two outliers; see Materials and Methods). During application of fields, neuron coherence (as measured by the Rayleigh vector strength) increased with the mean firing phase falling approximately within one-quarter of the oscillatory cycle $\left(40^{\circ}-110^{\circ}\right)$ (Fig. 3 ). For each cell, the coherence and mean phase were plotted against the field amplitude applied to that cell (in millivolts per millimeter) times the individual cell susceptibility (determined as above using interleaved DC stimulation trials) and divided by the current-induced voltage ramp slope and field period [i.e., $c E(\dot{V} T)^{-1}$; see Discussion]; the resulting 
plots (Fig. 3C) show significant correlation with this characteristic parameter (phase, $p<0.02$; coherence, $p<0.002$ ).

\section{Discussion}

\section{Predictions of timing changes}

Despite mounting phenomenological evidence that small fields can entrain network activity and have an effect on brain function, to date, there is no experimentally verified mechanistic theory on how this causal interaction may occur. We hypothesized that incremental polarization attributable to an extracellular field will affect the time of threshold crossing; under the assumption of a constant firing threshold, we made a number of predictions that will now be described.

For the case of low-frequency or DC extracellular fields with strength $E$, which polarize a cell by $\Delta V_{E}=c E$, where $c$ is the susceptibility of the membrane to an extracellular field, we predicted an advance in spike timing of the following:

$$
\Delta t=\frac{\Delta V_{E}}{\dot{V}_{I}}=\frac{c E}{\dot{V}_{1}} .
$$

Note that $c$ refers to the susceptibility effective at the site of spike initiation (i.e., the soma). Subscripts emphasize that $\Delta V_{E}$ results from application of the extracellular field, whereas $\dot{V}_{I}$ is the result of a concurrent intracellular/synaptic current ramp that induces the action potential spike.

For the case of AC fields, we predicted increased coherence of spiking with the ongoing oscillations. Change in coherence can be quantified as a change in the distribution of firing times occurring at phase $\varphi$ within the oscillatory cycle (with duration $T$ ). The distribution of firing phase, $p(\varphi)$, for the present experimental preparation can be expressed as follows:

$$
p(\varphi) \propto \frac{1}{2 \pi}+\frac{1}{T} \frac{c E}{\dot{V}_{I}} \cos (\varphi+\alpha),
$$

assuming that $\varphi$ is within a depolarizing phase of the membrane oscillation and the cell has not yet fired (Fig. $3 B$, green shaded region); otherwise, $p(\varphi)=0$, because only the time to the first spike was analyzed in the experiment (red shaded region). Equation 3 assumes a uniform initial distribution and no noise (for deviation, see supplemental data, available at www.jneurosci.org as supplemental material). Because the membrane acts as a lowpass filter, a delay between the extracellular field and the induced transmembrane polarization phase is expected; this is expressed in Equation 3 as phase delay, $\alpha$. The phase distribution of Equation 3 can be characterized by the mean phase, $\Phi$, and the vector strength, $r$, as shown in Figure $3 C$. Both should increase monotonically with $c E / \dot{V}$, with mean phase remaining constrained to the range, $\alpha \leq \Phi \leq \alpha+90^{\circ}$ (supplemental Fig. S1, available at www.jneurosci.org as supplemental material).

This series of predictions were born under the experimental conditions tested.

For both DC and AC fields, the strength of the effect on spike timing scales with

$$
\frac{c E}{\dot{V}}=G E,
$$

where $G=c / \dot{V}$ can be considered as a timing amplification factor. It increases with the susceptibility of the membrane to extracellular polarization (millivolts of transmembrane polarization at the site of action potential initiation per millivolts per millimeter extracellular field) and decreases with the depolarizing synaptic ramp slope (in millivolts per second). For the case of $\mathrm{AC}$ fields, the timing amplification factor is further scaled by field frequency $(1 / T)$.

\section{Generalization and quantitative parameterization}

Equations 2-4 provide a general theoretical framework for considering the effects of any electric field on spike timing in any neuron with appropriate membrane dynamics (see below). Quantitative application requires knowledge of the intracellular (ramp slope) and extracellular (field amplitude and period) waveforms, which are readily measured. For low-frequency (quasi-static) fields, cell susceptibility can be determined using the method outlined above.

Equation 3 can be readily applied to any periodic field with a complex spatiotemporal profile by considering only the maximum positive instantaneous $c E$ (the phase in the field cycle when the induced transmembrane depolarization is maximal). The susceptibility, $c$, is specific to a given spatiotemporal waveform (e.g., frequency, nonuniformity). The uniform field susceptibility, for any given temporal waveform, presumably sets a lower susceptibility bound for nonuniform fields. The membrane, acting as a low-pass field filter (Bikson et al., 2004) may introduce a frequency-specific phase delay $\alpha>0^{\circ}$, as well as an attenuated (relative to DC) susceptibility. The DC field susceptibility thus sets an upper limit for AC fields of comparable spatial profile. The phase delay may be estimated assuming the membrane acts as a first-order low-pass filter; thus, a membrane time constant of $\tau=30 \mathrm{~ms}$ should result in a phase delay of $\alpha \approx 40^{\circ}$ for $30 \mathrm{~Hz}$ fields, consistent with our experimental findings using timing data (Fig. 3).

\section{Applying timing changes to discern susceptibility}

As reinforced above, the polarization susceptibility of neurons is of fundamental importance in assessing subsequent effects on nervous system function. The field-induced polarization is inherently variable between neurons attributable in part to alignment with the field, neuronal geometry, and membrane biophysical properties (Rattay, 1998). Here we developed a method using measured timing changes to calculate the sensitivity of the neuron to polarization by an electric field (Fig. 2B). This technique may yield greater accuracy in measuring polarization sensitivity to small $(<5 \mathrm{mV} / \mathrm{mm})$ electric fields than can be achieved by conventional methods. Conventional methods are limited because experimental (line) noise is of magnitude comparable with the polarization induced by small fields, and they require an additional, precisely positioned, isopotential field electrode. Our timing-based method may be readily applied for nonuniform fields (e.g., unipolar, bipolar, deep brain stimulation spatial waveforms) and in vivo. As an additional advantage, our timing method may inherently extract the susceptibility at the spike initiation zone. Conventional polarization measurements used here and in previous studies (Bikson et al., 2004) show average susceptibility (coupling constant) values similar to our method using timing changes.

\section{Membrane dynamics and noise}

The theory developed here assumes a simple threshold mechanism equivalent to an integrate-and-fire model neuron with constant firing threshold. The accuracy of the assumption of constant threshold depends on a number of factors, including the channel kinetics of the specific cell type and the nature of the incoming synaptic input; experimental and theoretical work show both dynamic and constant thresholds (Fricker et al., 1999; Azouz and Gray, 2000). The present experiments with DC stim- 
ulation controlled for potential slope-dependent thresholds by measuring only changes in timing relative to a control condition with the same ramp slope. The experimental data for the AC stimulation is generally consistent with a constant threshold.

Interestingly, our theory on the scaling of timing effects with ramp slope also explains a number of existing observations on the timing jitter that results from synaptic and membrane noise. If noise induces variability in the membrane potential $\left(\Delta V_{n}\right)$, we expect that the resulting spike timing jitter will scale with $\Delta t=$ $\Delta V_{n} / \dot{V}$. Indeed, under frozen-noise stimulation, precise spike timing is observed (Bryant and Segundo, 1976), with a timing precision that increases for faster stimuli (Mainen and Sejnowski, 1995). Corresponding simulations with a Hodgkin-Huxley neuron subject to membrane noise also show that increased stimulus magnitude increases timing precision (Schneidman et al., 1998). A similar inverse dependence of spike time precision with stimulus intensity can be observed in cortical pyramidal neurons subject to synaptic noise (Shu et al., 2003). According to our theory, both a reduced time constant and an increased magnitude of the stimulus will increase membrane ramp slope and thus reduce timing jitter attributable to noise.

\section{Functional amplification: network consequences}

We found that a $1 \mathrm{mV} / \mathrm{mm}$ uniform field induced on average a transmembrane potential change of $\sim 0.1 \mathrm{mV}$. Compared with the scale of depolarization necessary to bring a neuron from rest to threshold $(\sim 15 \mathrm{mV})$, these fields were previously considered insignificant with respect to action potential initiation. Previous action potential threshold studies identified changes attributable to electric fields of $<5 \mathrm{mV} / \mathrm{mm}$ (Jefferys, 1981). Rather than spike generation, here we demonstrated changes in timing, consistent with the proposed amplification mechanism. The present results provide a potential mechanism for the effects on network spike timing demonstrated previously in vitro with exogenous uniform fields as low as $0.1 \mathrm{mV} / \mathrm{mm}$ (Deans et al., 2003; Francis et al., 2003; Fujisawa et al., 2004) and in vivo with calculated fields of 1.2 $\mathrm{mV} / \mathrm{mm}$ (Marshall et al., 2006).

Electric field-induced changes in spike timing would be particularly relevant for temporal coding during coherent (synchronous) network activity. For example, theta-modulated gamma activity in the hippocampus has been identified as a physiological correlate for a number of phenomena, including spatial navigation, memory, etc. (Miller, 1991; Kahana et al., 2001; Canolty et al., 2006). Extracellular gamma activity amplitudes of up to 0.2 $\mathrm{mV}$ are observed in vivo (Csicsvari et al., 2003) and in vitro (Hajos et al., 2004; Mann et al., 2005), with complete phase reversal across $100 \mu \mathrm{m}$ (from stratum pyramidale to stratum radiatum) resulting in field gradients of $4 \mathrm{mV} / \mathrm{mm}$. An estimated gamma field-induced polarization of at least $\Delta V_{E}=0.4 \mathrm{mV}$ (uniform field sensitivity providing a lower bound for the coupling factor) is small compared with the full-scale voltage fluctuations (e.g., AP threshold); however, considering a theta oscillation synaptic ramp with slope of $\dot{V}_{I}=0.2 \mathrm{mV} / \mathrm{ms}$ ( $15 \mathrm{mV}$ change between resting and firing potentials within $75 \mathrm{~ms}$ of half a theta cycle), a time shift of $\Delta t=1 \mathrm{~ms}$, and a Rayleigh vector strength of $r=0.39$ with a mean angle of $\alpha$ can be estimated. Thus, extracellular fields oscillating at gamma frequency may have a functional effect on AP timing during theta activity even in a single cycle. Moreover, because gamma field activity is coherent across several millimeters of the pyramidal layer (Csicsvari et al., 2003; Mann et al., 2005), extracellular gamma oscillations could facilitate coherence across a large population of neurons. Finally, computational studies have demonstrated that an addi- tional synchronizing effect may result during recurrent network activity when small changes in time add up over multiple cycles (Parra and Bikson, 2004).

The discussion above assumes that the timing of extracellular fields relative to synaptic input acts to promote coherent firing by advancing late spikes and delaying early spikes. However, a different temporal relationship between fields and underlying network activity could also have the opposite effect of advancing early spikes and delaying late spikes, thus acting as a safety mechanism to prevent hypersynchronization.

Our approach for predicting field-induced timing changes (Eqs. 2, 3) can be readily extended to other networks using readily measurable extracellular and intracellular waveforms and using either field susceptibility estimates or our novel method to measure susceptibility. Indeed, extracellular field oscillations are a ubiquitous marker for network oscillations (Chrobak and Buzsaki, 1998; Donoghue et al., 1998, Csicsvari et al., 2003; Nunez and Srinivasan, 2005; Sarnthein and Jeanmonod, 2007). More than an epiphenomenon, our results indicate a functional role for field potentials in modulating spike timing. Indeed, the information content of endogenous extracellular potential oscillations is often comparable with multiunit activity (Scherberger et al., 2005; Heldman et al., 2006; Liu and Newsome, 2006). Synaptic mechanisms underlie the generation of many classes of oscillations (Bragin et al., 1995; McBain et al., 1999; Buzsaki et al., 2003; Jones and Wilson, 2005; Netoff et al., 2005; Siapas et al., 2005); field-effect coupling is not proposed as alternative but rather as a complementary mechanism with unique spatiotemporal features.

Our system does not presuppose the source, exogenous or endogenous, of the modulating electric fields. Thus, the equations developed in this paper can be similarly applied in predicting the effects of environmental electric fields; electromagnetic field safety standards have not previously considered effects of timing. Finally, our results support the development of therapeutic stimulation technologies targeting neuronal timing; abnormal timing is indeed a hallmark of many neurological disorders, and subthreshold stimulation approaches may be readily adapted for non-invasive (transcranial) technologies.

\section{References}

Azouz R, Gray CM (2000) Dynamic spike threshold reveals a mechanism for synaptic coincidence detection in cortical neurons in vivo. Proc Natl Acad Sci USA 97:8110-8115.

Bikson M, Inoue M, Akiyama H, Deans JK, Fox JE, Miyakawa H, Jefferys JG (2004) Effects of uniform extracellular DC electric fields on excitability in rat hippocampal slices in vitro. J Physiol (Lond) 557:175-190.

Bragin A, Jando G, Nadasdy Z, Hetke K, Wise K, Buzsaki G (1995) Gamma $(40-100 \mathrm{~Hz})$ oscillation in the hippocampus of the behaving rat. J Neurosci 15:47-60.

Bryant HL, Segundo JP (1976) Spike initiation by transmembrane current: a white-noise analysis. J Physiol (Lond) 260:279-314.

Buzsaki G, Buhl DL, Harris KD, Csicsvari J, Czeh B, Morozov A (2003) Hippocampal network patterns of activity in the mouse. Neuroscience 116:201-211.

Canolty RT, Edwards E, Dalal SS Soltani M NAgajaran SS, Kirsch HE, Berger MS, Barbaro NM Knight RT (2006) High gamma power is phase-locked to theta oscillations in human neocortex. Science 313:1626-1628.

Chan CY, Nicholson CJ (1986) Modulation by applied electric fields of Purkinje and stellate cell activity in the isolated turtle cerebellum. J Physiol (Lond) 371:89-114.

Chrobak JJ, Buzsaki G (1998) Gamma oscillations in the entorhinal cortex of the freely behaving rat. J Neurosci 18:388-398.

Chrobak JJ, Lorincz A, Buzsaki G (2000) Physiological patterns in the hippocampo-entorhinal cortex system. Hippocampus 10:457-465.

Csicsvari J, Jamieson B, Wise KD, Buzsaki G (2003) Mechanisms of gamma oscillations in the hippocampus of the behaving rat. Neuron 37:311-322. 
de Ruyter van Steveninck RR, Lewen GD, Strong SP, Koberle R, Bialek W (1997) Reproducibility and variability in neural spike trains. Science 275:1805-1808.

Deans JK, Bikson M, Fox JE, Jefferys JGR (2003) Effects of AC fields at powerline frequencies on gamma oscillations in vitro. Soc Neurosci Abstr 29:258.1.

DeWeese MR, Wehr M, Zador AM (2003) Binary spiking in auditory cortex. J Neurosci 23:7940-7949.

Donoghue JP, Sanes JN, Hatsopoulos NG, Gaál G (1998) Neural discharge and local field potential oscillations in primate motor cortex during voluntary movements. J Neurophysiol 79:159-173.

Durand DM, Bikson M (2001) Suppression and control of epileptiform activity by electrical stimulation: a review. Proc IEEE 89:1065-1082.

Fischer Y, Wittner L, Freund TF, Gahwiler BH (2002) Simultaneous activation of gamma and theta network oscillations in rat hippocampal slice cultures. J Physiol (Lond) 539:857-868.

Francis JT, Gluckman BJ, Schiff SJ (2003) Sensitivity of neurons to weak electric fields. J Neurosci 23:7255-7261.

Fricker D, Verheugen J, Miles R (1999) Cell-attached measurements of the firing threshold of rat hippocampal neurones. J Physiol (Lond) 517:791-804.

Fujisawa S, Matsuki N, Ikegaya Y (2004) Chronometric readout from a memory trace: gamma-frequency field stimulation recruits timed recurrent activity in the rat CA3 network. J Physiol (Lond) 561:123-131.

Ghai RS, Bikson M, Durand DM (2000) Effects of applied electric fields on low-calcium epileptiform activity in the CA1 region of rat hippocampal slices. J Neurophysiol 84:274-280.

Hajos N, Palhalmi J, Mann EO, Nemeth B, Paulsen O, Freund TF (2004) Spike timing of distinct types of GABAergic interneuron during hippocampal gamma oscillations in vitro. J Neurosci 24:9127-9137.

Hamblin DL (2002) Effects of mobile phone emissions on human brain activity and sleep variables. Int J Radiat Biol 78:659-669.

Harris K, Henze D, Hirase H, Leinekugel X, Dragol G, Czurko A, Buzsaki G (2002) Spike train dynamics predicts theta-related phase precession in hippocampal pyramidal cells. Nature 417:738-741.

Harris KD, Csicsvari J, Hirase H, Dragoi G, Buzsaki G (2003) Organization of cell assemblies in the hippocampus. Nature 424:552-556.

Heldman DA, Wang W, Chan SS, Moran DW (2006) Local field potential spectral tuning in motor cortex during reaching. IEEE Trans Neural Syst Rehabil Eng 14:180-183.

Jefferys JG (1981) Influence of electric fields on the excitability of granule cells in guinea-pig hippocampal slices. J Physiol (Lond) 319:143-152.

Jefferys JG, Deans J, Bikson M, Fox J (2003) Effects of weak electric fields on the activity of neurons and neuronal networks. Radiat Prot Dosimetry 106:321-323

Jones M, Wilson M (2005) Theta rhythms coordinate hippocampalprefrontal interactions in a spatial memory task. PLOS Biology 3:e402.

Kahana M, Seelig J, Madsen JR (2001) Theta returns. Curr Opin Neurobiol 11:739-744.

Kara P, Reinagel P, Reid RC (2000) Low response variability in simultaneously recorded retinal, thalamic, and cortical neurons. Neuron 27:635-646.

Kashiwadani H, Sasaki YF, Uchida N, Mori K (1999) Synchronized oscillatory discharges of mitral/tufted cells with different molecular receptive ranges in the rabbit olfactory bulb. J Neurophysiol 82:1786-1792.

Liu J, Newsome WT (2006) Local field potential in cortical area MT: stimulus tuning and behavioral correlations. J Neurosci 26:7779-7790.

Lutz A, Lachaux JP, Martinerie J, Varela F (2001) Guiding the study of brain dynamics by using first person data: synchrony patterns correlate with ongoing conscious states during a simple visual task. Proc Natl Acad Sci USA 99:1586-1591.

Mainen ZF, Sejnowski TJ (1995) Reliability of spike timing in neocortical neurons. Science 268:1503-1506.

Mainen ZF, Sejnowski TJ (1996) Influence of dendritic structure on firing pattern in model neocortical neurons. Nature 382:363-366.

Mann EO, Radcliffe CA, Paulsen O (2005) Hippocampal gamma-frequency oscillations: from interneurones to pyramidal cells, and back. J Physiol (Lond) 562:55-63.

Marshall L, Helgadottir H, Molle M, Born J (2006) Boosting slow oscillations during sleep potentiates memory. Nature 444:610-613.

McBain C, Freund T, Mody I (1999) Glutamatergic synapses onto hippocampal interneurons: precision timing without lasting plasticity. Trends Neurosci 22:228-235.

Mehta MR, Lee AK, Wilson MA (2002) Role of experience and oscillations in transforming a rate code into a temporal code. Nature 417:741-746.

Miller R (1991) Cortico-hippocampal interplay and the representation of contexts in the brain. New York: Springer.

Moore DS, McCabe GP (1999) Introduction to the practice of statistics, Ed 3. New York: Freeman.

Netoff T, Banks M, Dorval A, Acker C, Haas J, Kopell N, White J (2005) Synchronizationn I hybrid neuronal networks of the hippocampal formation. J Neurophysiol 93:1197-1208.

Nunez PL, Srinivasan RS (2005) Electric fields of the brain: the neurophysics of EEG. Oxford: Oxford UP.

Parra LC, Bikson M (2004) Model of the effect of extracellular fields on spike time coherence, 26th Annual International Conference of the IEEE Engineering in Medicine and Biology Society, San Francisco, CA, September.

Radman T, Parra LC, Bikson M (2006) Amplification of small electric fields by neurons; implications for spike timing, 28th Annual International Conference of the IEEE Engineering in Medicine and Biology Society, New York, NY, August-September.

Rattay F (1998) Analysis of the electrical excitation of CNS neurons. IEEE Trans Biomed Eng 45:766-772.

Reinagel P, Reid RC (2000) Temporal coding of visual information in the thalamus. J Neurosci 20:5392-5400.

Riehle A, Grammont F, Diesmann M, Grun S (2000) Dynamical changes and temporal precision of synchronized spiking activity in monkey motor cortex during movement preparation. J Physiol (Paris) 94:569-582.

Sarnthein J, Jeanmonod D (2007) High thalamocortical theta coherence in patients with Parkinson's disease. J Neurosci 27:124-131.

Schaefer AT, Angelo K, Hartwig S, Margrie T (2006) Neuronal oscillations enhance stimulus discrimination by ensuring action potential precision. PLoS Biol 4:1010-1024.

Scherberger H, Jarvis MR, Andersen RA (2005) Cortical local field potential encodes movement intentions in the posterior parietal cortex. Neuron 46:347-354.

Schneidman E, Freedman B, Segev I (1998) Ion channel stochasticity may be critical in determining the reliability and precision of spike timing. Neural Comput 10:1679-1703.

Shu Y, Hasenstaub A, Badoual M, Bal T, McCormick DA (2003) Barrages of synaptic activity control the gain and sensitivity of cortical neurons. J Neurosci 23:10388-10401.

Siapas AG, Lubenov EV, Wilson MA (2005) Prefrontal phase locking to hippocampal theta oscillations. Neuron 46:141-151.

Trussell LO (1999) Synaptic mechanisms for coding timing in auditory neurons. Annu Rev Physiol 61:477-496.

Webster BR, Celnik PA, Cohen LG (2006) Noninvasive brain stimulation in stroke rehabilitation. NeuroRx 3:474-481. 